\title{
The Growth of Mouse Hybridoma Cells between Peyer's Patch Lymphocyte and Myeloma Cell in the Gastrointestinal Tract. A Model for Human Multiple Lymphomatous Polyposis of the Gastrointestinal Tract
}

\author{
Kiyoo Kuroe, Ayako Yoshihara, Masahiro Akiyama, \\ Hiromitsu Takemori and Yutaka Yoshida \\ The First Department of Internal Medicine, Hirosaki \\ University School of Medicine, Hirosaki 036
}

\begin{abstract}
Kuroe, K., Yoshinara, A., Akiyama, M., Takemori, H., and Yoshida, Y. The Growth of Mouse Hybridoma Cells between Peyer's Patch Lymphocyte and Myeloma Cell in the Gastrointestinal Tract. A model for Human Multiple Lymphomatous Polyposis of the Gastrointestinal Tract. Tohoku J. Exp. Med., $1993,170(3), 197-205$ - To elucidate the mechanism of multiple lesions in human primary lymphoma or multiple lymphomatous polyposis of the gastrointestinal tract, we developed a experimental model utilizing mouse hybridoma cells. Syngeneic mice were immunized with human hemoglobin via gastric intubation and a hybridoma clone (13c) between the Peyer's patch cell and mouse myeloma cell was obtained. The $13 \mathrm{c}$ cells were infused into normal syngeneic mice through a lateral tail vein. About 2 months after injection mice were sacrificed and their viscera were scrutinized histologically. All 11 mice receiving 13c showed multiple tumors at the colon, microscopic massive invasions at the small intestine, and scattered invasions at Peyer's patch, mesenteric lymph nodes and the spleen, exhibiting the similar distribution patterns as in human multiple lymphomatous polyposis of the gastrointestinal tract. ME-1 or MU-1 cells (hybridomas between P3 and mouse spleen cell) were distributed at the liver, spleen and paraaortic lymph nodes. Such migration patterns of $13 \mathrm{c}$ cells and their adherence to vessel walls suggest that multiple invasions may be lymphocyte homing receptormediated phenomena. multiple lymphomatous polyposis of the gastrointestinal tract; gastrointestinal lymphoma; Peyer's patch; mouse hybridoma; lymphocyte homing
\end{abstract}

Primary lymphoma of the gastrointestinal tract is a rare disease and sometimes accompanies other multiple lesions along the length of the tract without invading peripheral lymphoid tissues. Multiple lymphomatous polyposis of the gastrointestinal tract is also found as a very rare case. This type of lymphoma is a primary disease in which all the submucosal lymphoid tissues are involved in lymphomatous proliferation. Although the mechanism of multiple lesions remains unknown, the following two possibilities might be considered; simultane-

Received May 25, 1993; revision accepted for publication June 13, 1993. 


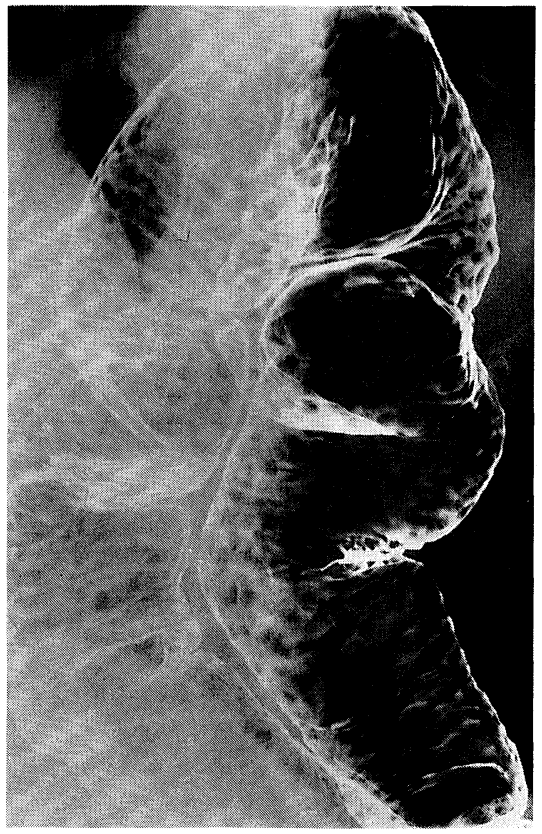

Fig. 1. Invasive polypoid lesions were seen in a patient with multiple lymphomatous polyposis of the gastrointestinal tract.

ous malignancies as multicentric origins or metastasis of malignant lymphoma cells from a primary lesion.

We recently experienced a case of multiple lymphomatous polyposis of the gastrointestinal tract. Barium contrast study of the colon showed infiltration of the bowel wall with polypoid lesions (Fig. 1). The location of invasive polypoid lesions in this patient was shown in Fig. 2. Those were distributed in the stomach, duodenum, small intestine and large intestine, but not in the liver or spleen. Several sizable masses projected into those lumina. This suggested that these multiple lesions may be due to migration of recirculating gut lymphoma cells via bloodstream to particular lymphoid organs rather than simultaneous malignancies as multicentric origins. In this paper, to examine the mechanism of these multifocal lesions, we established a hybridoma cell line between Peyer's patch cell and mouse myeloma cell, and infused these cells through a tail vein to the syngeneic recipient mice. A preliminary report of this method was described previously (Kuroe et al. 1987). The distribution of this hybridoma cell line was compared with those of P3 and other hybridoma cell lines, ME-1 and MU-1.

\section{Materials and Methods}

Animals. BALB/c mice were bred and maintained in our facilities. Eight to 10 week old female mice were used.

Cell line. P3X63. Ag8. $653 \mathrm{BALB} / \mathrm{c}$ mouse myeloma cells (P3) were routinely 


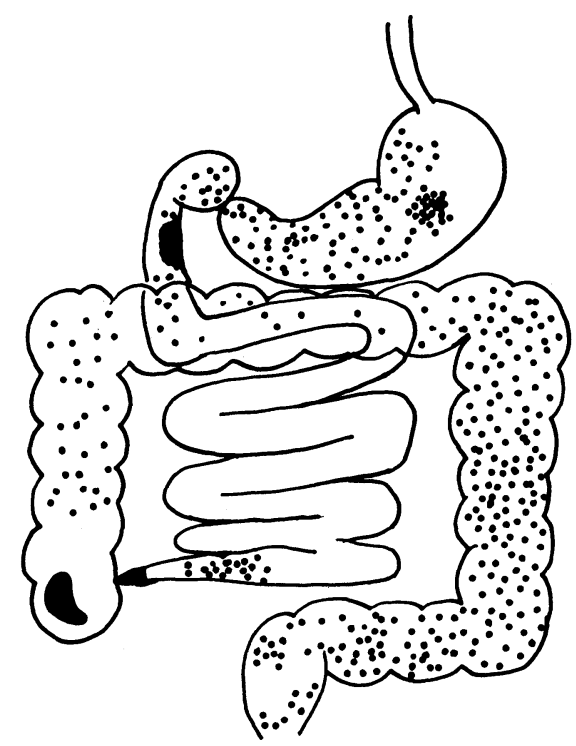

Fig. 2. Invasive polypoid lesions were distributed to the stomach, duodenum, small intestine, and large intestine. Several sizable masses projected into the lumen in the patient.

maintained in Dulbecco's modified Eagle medium (D-MEM; Gibco, Gaithersburg, MD, USA) supplemented with $10 \%$ fetal calf serum (FCS; Gibco). The fastest-growing 8-azaguanine (8-AG; Sigma, St. Louis, MO, USA)-resist clones were expanded, and after certifying to be hypoxanthine/amethopterin/thymidine (HAT; Sigma) sensitive, they were used for cell fusion with BALB/c mouse Peyer's patch cells two weeks after treatment with 8-AG. ME-1 and MU-1 are hybridoma cell lines fused between BALB/c mouse spleen cells and P3 myeloma cells, which we previously established (Akiyama et al. 1986).

Immunization and preparation of Peyer's patch cells. Inbred mice were given human hemoglobin via gastric intubation under ether anesthesia once a week 4 times. Four days after last oral immunization with human hemoglobin, Peyer's patches of 12 mice were aseptically cut out from the wall of the small intestine, and were washed repeatedly in Hank's balanced salt solution (HBSS) supplemented with an antibiotic solution and $10 \mathrm{mM}$ dithiothreitol (DTT; Gibco) for $30 \mathrm{~min}$. Thereafter, they were washed and minced with scissors in petri dishes containing D-MEM. Single cell suspension was obtained by gentle disruption of the tissues by squashing with a large rubber plug. Then the suspension was filtered through a 220-stainless steel mesh. The suspension was washed three times in D-MEM by centrifugation at $200 \mathrm{~g}$ for $5 \mathrm{~min}$ and resuspended in media.

Production of hybridomas. For fusion of immunized Peyer's patch cells with mouse myeloma cells, $1.3 \times 10^{7}$ myeloma cells and $6.9 \times 10^{7}$ Peyer's patch cells were washed 3 times respectively and mixed in D-MEM without FCS, and were fused in $0.5 \mathrm{ml}$ of $42.5 \%$ (wt/vol) polyethylene glycol 2,000 (Wako, Osaka) at $37^{\circ} \mathrm{C}$. After washing twice in warm D-MEM, they were suspended at a concentration of 10 cells per $\mathrm{ml}$ in D-MEM with $20 \% \mathrm{FCS}$, and seeded $0.2 \mathrm{ml}$ of cell suspension in microtiter plates with flat-bottom wells (Coster, Cambridge, MA, USA). The hybrids were selected by incubation in HAT medium. HAT resistant hybrids grew out within 8-14 days, but incubation in HAT medium was continued for at least 2 weeks. Screening for anti-human hemoglobin Ao antibody production was performed with ELISA. Cells producing anti-hemoglobin antibodies were cloned by limiting 
dilution. One of such clones, named 13c, was established, which had the similar growth rate and colony forming capacity in vitro with those of P3, ME-1 and MU-1.

Infusion of hybridoma cells to mice. The suspension of $13 \mathrm{c}$ cells $\left(3 \times 10^{6} / \mathrm{ml}\right)$ was injected into 11 recipient mice through a lateral tail vein once a week 2 times. Each suspension $\left(3 \times 10^{6} / \mathrm{ml}\right)$ of ME-1, MU-1 or P3 was also tested in the same manner. About 2 months after the injection, mice were sacrificed and their viscera were examined macroscopically, then subjected to histologic examination. Viscera were fixed at $10 \%$ buffered formalin and sections were stained with hematoxylin and eosin (H-E).

\section{RESULTS}

The 13c clone was a large cell with a polymorphous nucleus and coarse chromatins (Fig. 3), easily distinguishable from other cells in the sections of tissues stained with $\mathrm{H}-\mathrm{E}$. The distribution in the recipient mice has been studied by identifying these clone cells with the morphological characteristics. In all 11 mice injected $13 \mathrm{c}$ showed similar distribution patterns.

As shown in Table 1, there existed differences in pattern of invasion between 13c clone and ME-1, MU-1 or P3. The 13c formed multiple tumors at the colon, and showed microscopically massive invasions at the small intestine, scattered invasions at Peyer's patches, mesenteric lymph nodes and the spleen. It did not invade the liver and lung. Histologic examination revealed that the $13 \mathrm{c}$ migrated into lamina propria sparing lymphoid tissues (Fig. 4A, B) and formed massive invasions with destruction of lamina propria in the colon (Fig. $4 \mathrm{C}$ ) and small intestine. At the small intestine 13c proliferated in villi, and expanded and replaced them. The 13c which migrated into solitary lymphoid follicles, replaced them and formed multiple tumors at the colon (Fig. 5A, B). Fig. 6 shows distribution of 13c at the colon in one of 11 mice, exhibiting the similar distribution pattern as in human multiple lymphomatous polyposis of the gastrointestinal

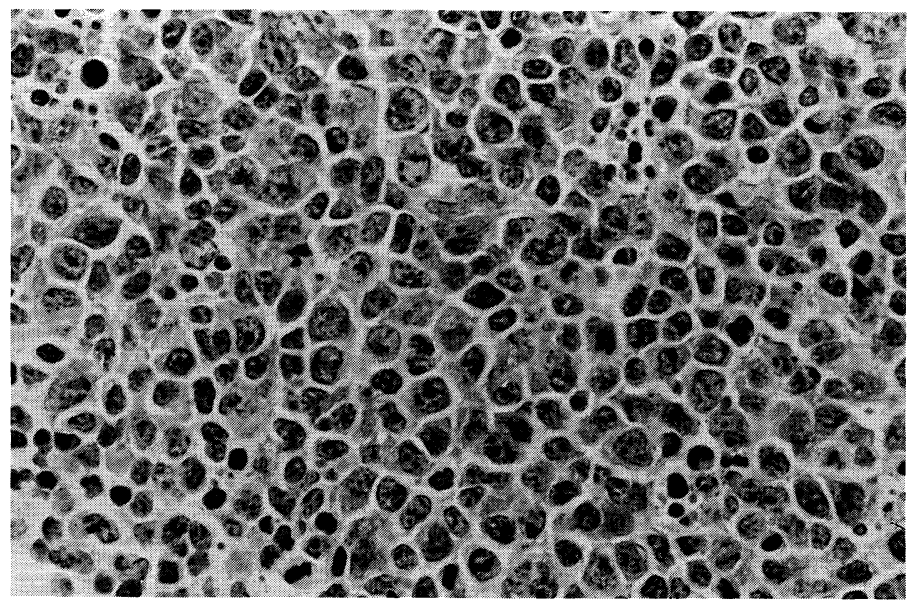

Fig. 3. 13c clone tumors consisted of large cells with polymorphous nuclei and coarse chromatin $(\times 400)$ 
TABLE 1. Locations of invasion of hybrids or myeloma cells injected into syngeneic recipient mice

\begin{tabular}{|c|c|c|c|c|}
\hline \multirow[t]{2}{*}{ Tissue } & \multirow{2}{*}{$\begin{array}{c}\text { Hybrid between } \\
\text { PP lymphocyte } \\
\text { and myeloma cell }\end{array}$} & \multicolumn{2}{|c|}{$\begin{array}{l}\text { Hybrid between } \\
\text { spleen lymphocyte } \\
\text { and myeloma cell }\end{array}$} & \multirow{2}{*}{$\frac{\text { Myeloma cell only }}{\text { P3 }}$} \\
\hline & & ME-1 & MU-1 & \\
\hline \multicolumn{5}{|l|}{ Gut } \\
\hline Colon & \# & - & - & - \\
\hline Small intestine & H & - & - & - \\
\hline Peyer's patch & + & - & - & - \\
\hline MLN & + & - & - & + \\
\hline PAL & - & + & \# & - \\
\hline Liver & - & \# & - & H \\
\hline Spleen & + & H & H & + \\
\hline Lung & - & - & - & - \\
\hline Bone marrow & - & & & H \\
\hline
\end{tabular}

MLN, mesenteric lymph node; PAL, para-aortic lymph node.

The grade of infiltrations of hybrid cells was expressed as following: $\mathrm{H}$, tumor formation; H, massive invasion which was observed microscopically; + , scattered invasion; -, no invasion.

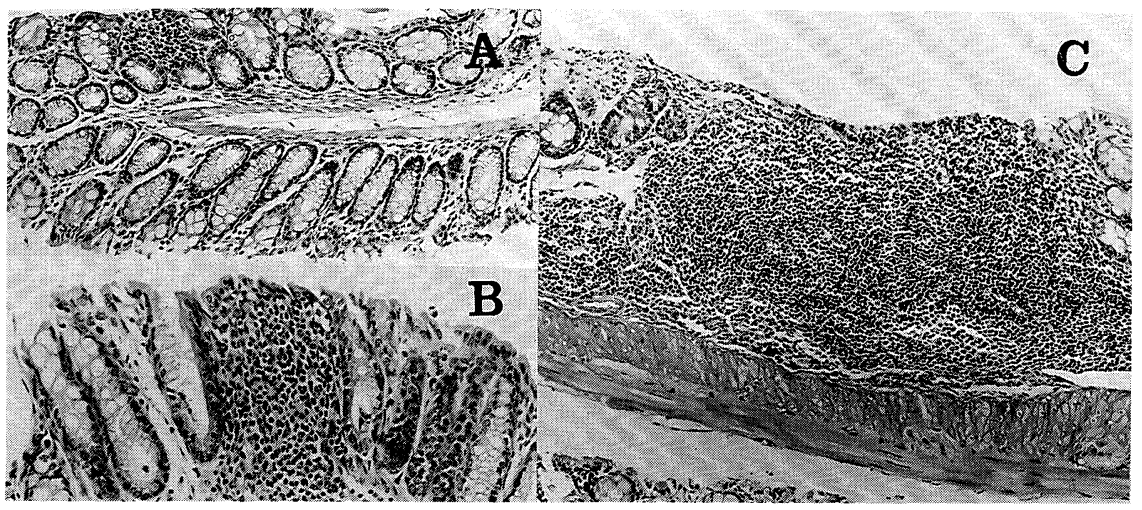

Fig. 4. 13c migrated into lamina propria of the gut sparing lymphoid tissue (A, $\mathrm{B} ; \times 100)$ and formed massive invasions with destruction of lamina propria $(\mathrm{C} ; \times 100)$.

tract. The 13c clone cells were found in vessel walls of the tumors and massive (Fig. 7A) and scattered invasion areas (Fig. 7B) at the colon.

Each ME-1 or MU-1, both of which are hybridoma cells between P3 and spleen cells, supposed to possess characters of spleen cells, was distributed in the liver, spleen and sporadically at para-aortic lymph nodes, and did not show migration or tumor formation at the gut, Peyer's patch or mesenteric lymph nodes 


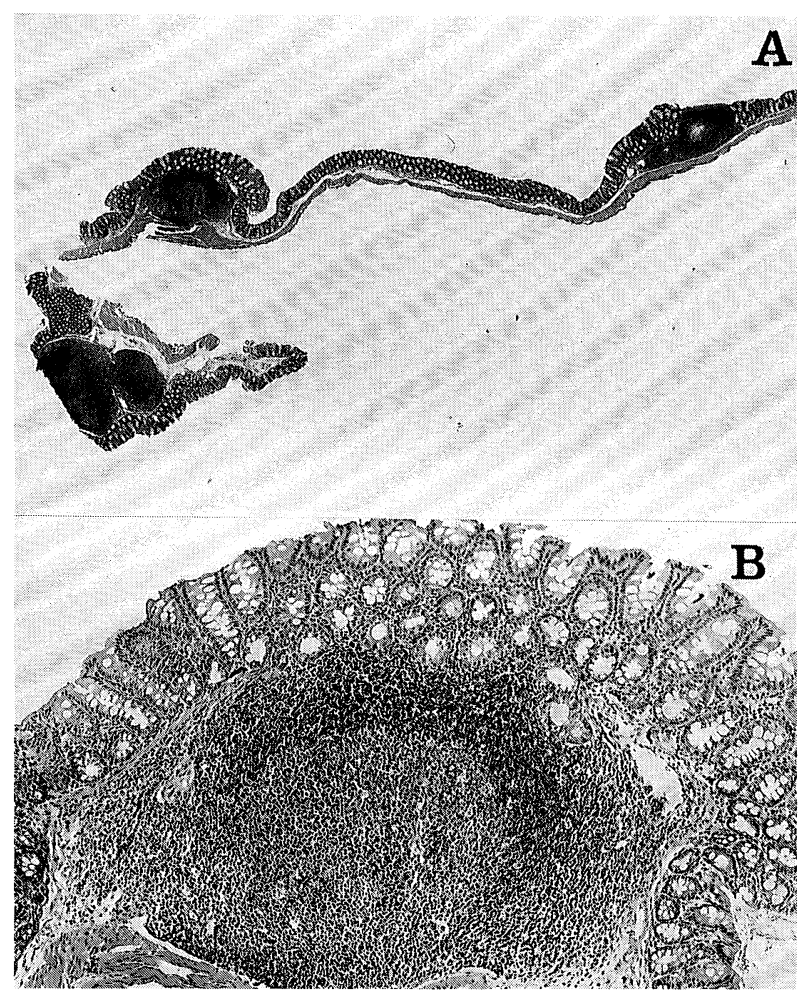

Fig. 5. 13c clone migrated into solitary lymphofollicles, and formed multiple tumors at the colon $(\mathrm{A} ; \times 20, \mathrm{~B} ; \times 100)$.

in 4 recipient mice. P3 did not migrate into the gut and Peyer's patch in 4 recipient mice. However, P3 cells were found in bone marrow, and sporadically in the spleen, mesenteric lymph nodes and para-aortic lymph nodes.

\section{Discussion}

It is recently known that most mature, small lymphocytes are constantly circulating, that is, leaving lymphoid organs in the efferent lymph, draining via the thoracic duct into the blood and then returning to other peripheral lymphoid organs. Lymphocytes leaving peripheral lymph nodes migrate preferentially to peripheral node, while lymphocytes leaving Peyer's patches migrate preferentially to gut-associated lymphoid tissues (GALT) (Griscelli et al. 1969; Guy-Grand et al. 1974; Chin et al. 1984). Gowans and Knight (1964) first defined lymph node entry sites to be postcapillary venules bearing high-walled endothelium; these vessels have subsequently been called high endothelial venules (HEV). It is recently known that lymphocyte homing is performed by a specific interaction between recirculating lymphocytes and lymphoid organ HEV (Butcher et al. 1980; Yamaguchi and Schoefl 1983). Lymphocyte surface structures for recognition of 


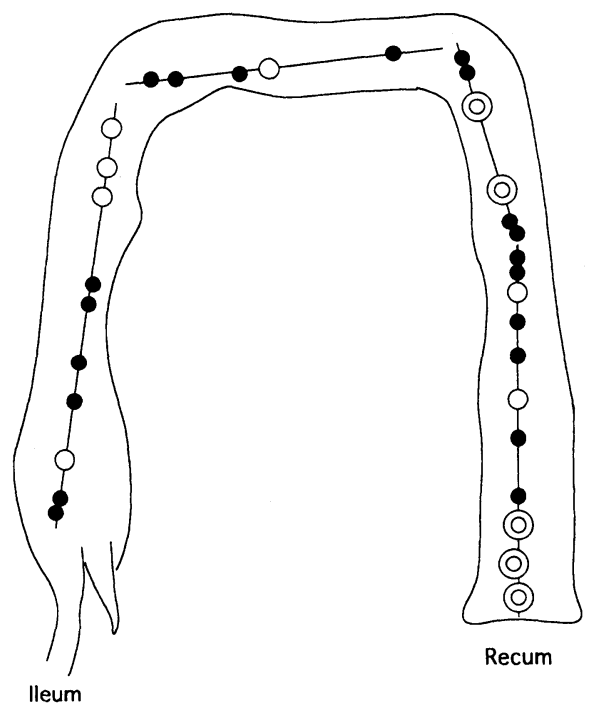

Fig. 6. Distribution of $13 \mathrm{c}$ clone at the colon. The specimen was histologically examined along the line. The grade of invasions was expressed as following: ( ) tumor; $\bigcirc$ massive invasion which was observed microscopically; $\bullet$ scattered invasion.

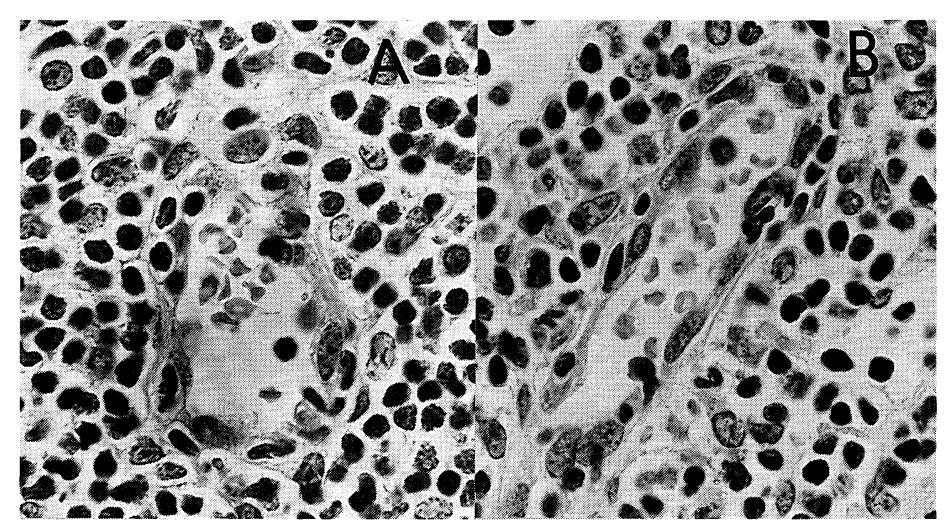

Fig. 7. 13c clone cells were found in the vessel wall of the massive $(A ; \times 400)$ or scattered invasion areas $(\mathrm{B} ; \times 400)$ at the colon.

and adherence to lymphoid organ $\mathrm{HEV}$ have been called homing receptors. Various homing receptors have been reported so far: Mel-14 (Gallatin et al. 1983; Siegelman et al. 1986), A.11 (Woodruf and Clarke 1987) and Leu8/TQ1 (Camerini et al. 1989) as lymphocyte molecules involved in binding to peripheral node $\mathrm{HEV}$, and 1.B2 (Woodruf and Clarke 1987), LPAM-1/VLA-4 (Holzmann et al. 1989) and Hermes-3 (Jalkanen et al. 1987), as those to mucosal HEV. After binding to these peculiar endothelial cells, lymphocytes transmigrate through the vessel wall into surrounding lymphoid parenchyma (Gowans and Knight 1964; Gutman and 
Weissman 1973). Mel 14 antigen is expressed on all peripheral node HEVbinding lymphomas and normal lymphocyte populations such as mesenteric node lymphocytes, pre-B cells, and thymocytes, but not on Peyer's patch HEV-binding or peripheral node non-HEV-binding lymphomas (Gallatin et al. 1983).

Lymphoma cells bearing homing receptors tend to metastasize to their cognate lymphoid organs via the bloodstream (Bargatze et al. 1987).

Peyer's patches are compact nodules of lymphoid cells that can readily be seen on the outer wall of the intestine and many of lymphoid cells can easily be obtained from Peyer's patches. This is a reason why we used Peyer's patch lymphocytes as an origin of gut lymphocytes and made the hybridoma. Peyer's patches contain mainly B lymphocytes that are committed to make a specialized form of immunoglobulin called IgA, and these B cells migrate from Peyer's patches (Craig and Cebra 1971; Husband and Gowans 1978) to epithelial surfaces lining the gut, mammary ducts, and salivary glands. The 13c clone producing antibody against human hemoglobin is supposed to have characters of gut B cell lymphoma.

The 13c migrated into lamina propria sparing lymphoid tissues and solitary lymphoid follicles of the gut, and formed massive invasions and tumors, espcially in the colon. Such migration patterns of $13 \mathrm{c}$ cells and their adherence to vessel walls suggest that multiple invasions may be homing-receptor-mediated phenomena. Formation of tumors may reflect enhanced proliferation of $13 \mathrm{c}$ cells in the colon in addition to the specific interaction via the homing receptors. ME-1 and MU-1, which are hybridomas between spleen lymphocyte and myeloma cell, or P3 did not migrate into the gut, presumably because these clones are devoid of homing receptors for the gut. Thirteen c, ME- 1 and MU- 1 clones are BALB/c mouse origin and no rejection was observed by intravenous injection into BALB/ c mice.

This experimental model may explain the formation of multiple lesions in human primary lymphoma of the gastrointestinal tract. Multiple lesions in human case may be also mediated by lymphocyte homing receptors. Lymphoma cells originating from human gut mucosa may be able to migrate to anywhere in human GALT via homing receptor as 13c clone in mouse experiments.

\section{References}

1) Akiyama, M., Takahashi, K., Yoshihara, A., Nikai, T., Murata, Y., Kuroe, K. \& Yoshida, Y. (1986) Monoclonal antibodies agaist human colonic mucosa. Digest. Organ Immunol., 16, 158-162. (in Japanese)

2) Bargatze, R.F., Wu, N.W., Weissman, I.L. \& Butcher, E.C. (1987) High endothelial venule binding as a predictor of the dissemination of passaged murine lymphomas. $J$. Exp. Med., 166, 1125-1131.

3) Butcher, E.C., Scollay, R.G. \& Weissman, I.L. (1980) Organ specificity of lymphocyte migration: Mediation by highly selective lymphocyte interaction with organ-specific determinants on high endothelial venules. Eur. J. Immunol., 10, 556- 
561.

4) Camerini, D., James, S.P., Stamenkovic, I. \& Seed, B. (1989) Leu-8/TQ1 is the human equivalent of the Mel-14 lymph node homing receptor. Nature, 342, 78-82.

5) Chin, Y., Rasmussen, R., Cakiroglu, A.G. \& Woodruff, J.J. (1984) Lymphocyte recognition of lymph node high endothelium. VI. Evidence of distinct structures mediating binding to high endothelial cells of lymph nodes and Peyer's patches. $J$. Immunol., 133, 2961-2965.

6) Craig, S.W. \& Cebra, J.J. (1971) Peyer's patches: An enriched source of precursors for IgA-producing immunocytes in the rabbit. J. Exp. Med., 134, 188-200.

7) Gallatin, W.M., Weissman, I.L. \& Butcher, E.C. (1983) A cell-surface molecule involved in organ specific homing of lymphocytes. Nature, 304, 30-34.

8) Gowans, J.L. \& Knight, E.J. (1964) The route of recirculation of lymphocytes in the rat. Proc. R. Soc. Lond. (Biol.), 159, 257-282.

9) Griscelli, C., Vassali, P. \& McCluskey, R.T. (1969) Distribution of large dividing lymph node cells in syngeneic recipient rats after intravenous injection. J. Exp. Med., 130, 1427-1452.

10) Gutman, G. \& Weissman, I.L. (1973) Homing properties of thymus-independent follicular lymphocytes. Transplantation, 16, 621-629.

11) Guy-Grand, D., Griscelli, C. \& Vassali, P. (1974) The gut-associated lymphoid system: Nature and properties of the large dividing cells. Eur. J. Immunol., 4, 435443.

12) Holzmann, B., Mclntyre, B.W. \& Weissman, I.L. (1989) Identification of a murine Peyer's patch-specific lymphocyte homing receptor as an integrin molecule with an $\alpha$ chain homologous to human VLA-4 $\alpha$. Cell, 56, 37-46.

13) Husband, A.J. \& Gowans, J.L. (1978) The origin and antigen-dependent distribution of IgA-containing cells in the intestine. J. Exp. Med., 148, 1146-1160.

14) Jalkanen, S., Bargatze, R.F., de los Toyos, J. \& Butcher, E.C. (1987) Lymphocyte recognition of high endothelium: Antibodies to distinct epitopes of an $85-95-\mathrm{KD}$ glycoprotein antigen differentially inhibit lymphocyte binding to lymph node, mucosal, or synovial endothelial cells. J. Cell. Biol., 105, 983-990.

15) Kuroe, K., Yoshihara, A., Takahashi, O., Nikai, T., Takahashi, K., Akiyama, M., Murata, Y. \& Yoshida, Y. (1987) Studies on homing of gut lymphocyte. Digest. Organ Immunol., 19, 17-21. (in Japanese)

16) Siegelman, M., Bond, M.W., Gallatin, W.M., St.John, T., Smith, H.T., Fried, V.A. \& Weisman, I.L. (1986) Cell surface molecule associated with lymphocyte homing is ubiquitinated branched-chain glycoprotein. Science, 231, 823-829.

17) Woodruf, J.J. \& Clarke, L.M. (1987) Specific cell-adhesion mechanisms determining migration pathways of recirculating lymphocytes. Annu. Rev. Immunol., 5, 201-222.

18) Yamaguchi, Y. \& Schoefl, G.I. (1983) Blood vessels of the Peyer's patch in the mouse: II. In vivo observations. Anat. Rec., 206, 403-417. 